\title{
Review
}

Jürgen Schiller, Katharina Lemmnitzer, Jan-Niklas Dürig and Jörg Rademann*

\section{Insights into structure, affinity, specificity, and function of GAG-protein interactions through the chemoenzymatic preparation of defined sulfated oligohyaluronans}

https://doi.org/10.1515/hsz-2021-0165

Received February 24, 2021; accepted July 4, 2021; published online July 22, 2021

\begin{abstract}
High amounts of glycosaminoglycans (GAG) such as hyaluronan (HA) occur in connective tissues. There is nowadays increasing evidence that a "sulfation code" exists which mediates numerous GAG functions. High molecular weight and inhomogeneity of GAG, however, aggravated detailed studies. Thus, synthetic oligosaccharides were urgently required. We will review here chemoenzymatic and analytic strategies to provide defined sulfated and anomerically modified GAG oligosaccharides of the HA type. Representative studies of protein/GAG interactions by (bio)chemical and biophysical methods are reported yielding novel insights into GAG-protein binding. Finally, the biological conclusions and in vivo applications of defined sulfated GAG oligosaccharides will be discussed.
\end{abstract}

Keywords: GAG oligosaccharides; GAG-protein binding; spectroscopic methods; structure-activity relationships; sulfation.

Correction note: Correction added after online publication July 22, 2021. Figure 2 has been updated in the article.

*Corresponding author: Jörg Rademann, Department of Biology, Chemistry, and Pharmacy, Institute of Pharmacy, Pharmaceutical and Medicinal Chemistry, Freie Universität Berlin, Königin-Luise-Str. 2+4, 14195 Berlin, Germany, E-mail: joerg.rademann@fu-berlin.de. https://orcid.org/0000-0001-6678-3165

Jürgen Schiller and Katharina Lemmnitzer, Faculty of Medicine, Institute of Medical Physics and Biophysics, University of Leipzig, Härtelstraße 16-18, 04107 Leipzig, Germany

Jan-Niklas Dürig, Department of Biology, Chemistry, and Pharmacy, Institute of Pharmacy, Pharmaceutical and Medicinal Chemistry, Freie Universität Berlin, Königin-Luise-Str. 2+4, 14195 Berlin, Germany

\section{Introduction}

Life expectancy and, thus, the number of patients suffering from many different diseases and delayed healing is continuously increasing. This poses a severe challenge to the health care system and leads to an increased need for tools to promote wound healing and tissue regeneration (Schnabelrauch et al. 2013). Sulfated glycosaminoglycans (GAG) were recognized as major components of the extracellular matrix in higher organisms, at the outer cell membrane of suspension cells and at tissue boundaries such as epithelial cells decades ago. As such, GAG exert numerous biological functions in processes such as cell growth, blood coagulation, immune reaction, infection and wound healing, which all are mediated by GAG-protein binding (Schnabelrauch et al. 2013). In consequence, many diverse proteins including growth factors, cytokines, proteolytic enzymes and further regulatory proteins display GAG-binding domains and the activities and local concentrations of these proteins are regulated by GAG-protein interactions. Few of these interactions have been studied in detail, for instance, the binding of heparin to proteins involved in the blood coagulation cascade such as antithrombin. Beyond heparins, which were introduced as potent anti-coagulants several decades ago, for a long time the knowledge of protein-GAG interactions has been limited and it took decades to discover how common GAG binding is among the proteins of the extracellular matrix.

These discoveries have raised the interest in synthetically modified sulfated GAG oligomers as such molecules can be valuable as chemical tools for studying and understanding the functional relevance, the structural biology, and the future application potential of GAG-protein interactions. Therefore, there is a high demand for the development of chemical methods that allow the flexible access to anomerically labeled, defined sulfated GAG. Typical labels of interest include fluorophores, which enable binding assay of GAG to protein. 
Finally, labeling methods for sulfated GAG will enable the systematic optimization of GAG to improve the affinity and possibly the specificity of protein binding and the modulation of GAG protein interactions in biochemical assays, in cells and finally in animal experiments.

\section{Why is the synthesis of defined GAG oligosaccharides challenging?}

Classically, oligosaccharides are synthesized using fully protected carbohydrate building blocks activated in the anomeric position as glycosyl donors. Glycosyl donors upon activation furnish glycosyl cations as reactive intermediates for the glycosylation of glycosyl acceptor molecules. Typical glycosyl donors include anomeric halogenides such as glycosyl bromides, chlorides, and fluorides, glycosyl phosphates, glycosyl thiols, and trichloro-acetimidates. Activation of glycosyl donors requires Lewis acids, oxidants, or alkylating agents. Glycosylation reactions require strictly anhydrous conditions and laborious, multi-step protocols for generating the regioselectively protected building blocks. The total synthesis of GAG oligosaccharides with defined sulfation patterns is time-consuming and results in poor yields due to the many involved steps. While the first synthesis of the octa-sulfo-pentaheparin "Fondaparinux" was published already in 1987 by Petitou and coworkers, the total synthesis of linear GAG such as chondroitin or hyaluronan was exclusively reported for the non-sulfated oligosaccharides (Vibert et al. 2009) or for chondroitin sulfates still carrying protecting groups (Eller et al. 2013). In consequence, total synthesis of defined sulfated GAG has not been useful to provide anomerically modified and defined sulfated GAG in quantities useful for biological studies, yet.

Therefore, we developed an alternative chemoenzymatic strategy to provide GAG oligosaccharides in gram quantities for subsequent modification (Köhling et al. 2016a). This strategy relies on the enzymatic degradation of the GAG polymer hyaluronic acid using commercially available hyaluronidases followed by size exclusion chromatography. By adaptation of the reaction and isolation protocols to the respective products, bovine testis hyaluronidase provided the tetra-hyaluronan HA4 with maximal isolated yields of $55-71 \%$ (Köhling et al. 2016a), the hexaoligosaccharide HA6 with 23\%, and the disaccharide HA2 with $11 \%$ yield (Köhling et al. 2016b). Oligohyaluronans dehydrated at the terminal glucuronic acid residue were obtained with the hyaluronidase from streptococcus pyogenes including the octasaccharide $\mathbf{\Delta H A 8}$ as well as $\Delta$ HA6 and $\Delta$ HA4 in yields of 28,23 , and $45 \%$, respectively
(Köhling et al. 2016b) for HA4, and with chemical methods that allow the conversion of unprotected sugars into anomerically modified GAG oligosaccharides.

\section{Selected synthetic strategies to provide anomerically modified GAG oligosaccharides with defined sulfation patterns}

\section{Anomeric modification}

Anomeric modification of unprotected and reducing GAG oligosaccharides is crucial to obtain chemically stable, pure diastereomers, which subsequently can be selectively sulfated and labeled to furnish the desired chemical tools. Traditionally, oligosaccharides are stabilized as $O$-glycosides, for example $O$-methyl glycosides, which are formed by treatment of the reducing sugar with acid in methanol. An early method to obtain anomerically pure and stable saccharides, which can be used for further derivatization was the conversion of sugar hemiacetals to 1-aminomodified sugars in ammonium carbonate buffer. The intermediary 1-amino sugars, however, are formed under basic conditions in only moderate yields after prolonged reaction time and need to be converted by $N$-acylation of the primary amino group to furnish chemically stable $\mathrm{N}$-glycoside products. Thus, we investigated several alternative protocols to provide stable, anomerically fixed and pure GAG oligosaccharides (Köhling et al. 2016a). The reaction with hydrazine produced sugar hydrazines, which were acylated to acylhydrazides with acceptable yields, however, the chemical stability of the products was insufficient at acidic $\mathrm{pH}$ and flexible anomeric modification was not possible. The activation of reducing sugar aldehydes with 2-chloro$N, N^{\prime}$-dimethyl-imidazolinium chloride (DMC), a method originally introduced by Tanaka et al. (2009) could be employed successfully for the anomeric modification of GAG oligosaccharides (Figure 1(b)) (Köhling et al. 2016a). Treatment of unprotected hyaluronan tetrasaccharide HA4, for example, with DMC and $N$-methylmorpholine as base in the presence of sodium azide furnished the anomeric azide, HA4-azide, in $72 \%$ yield and could be also employed to di-, and hexasaccharides HA2 and HA6. HA4- and HA6-azides proved to be valuable starting materials for the generation of diverse collections of defined sulfated, anomerically modified GAG employing the copper-assisted azide-alkyne couplings (CuAAC) furnishing anomeric 1,2,3-triazoles (Figure 1(e)). For cases in which the anomeric 1,2,3-triazole 
or the use of copper was not acceptable, we developed the first synthesis of unprotected 1-thio-glycosides enabling an alternative click reaction for GAG (Figure 1(c)) (Köhling et al. 2016b). For this, unprotected GAG like HA4, were activated with DMC and a thio-carboxylic acid such as thio-acetic acid or thio-benzoic acid was added, furnishing glycosyl1-thioesters. These stable and storable intermediates released under basic conditions the reactive hyaluronan1-thiols HA2, HA4-, and HA6-thiol, which reacted smoothly in thiol-click reactions such as Michael additions and thiol-ene reactions. These thiol-click reactions proved to be powerful for the synthesis of GAG-protein conjugates (Figure 1(f)) (Exner et al. 2016; Köhling et al. 2016b).

\section{Defined sulfation}

For defined sulfation protocols, the different reactivities of the GAG-hydroxy-groups could be exploited (Figure 1(d)). The challenge in selective sulfation reactions is the lability of GAG-sulfates under basic and acidic conditions; thus, only few reaction conditions are tolerated without sulfate loss or migration. Selective sulfation of the primary 6-OH groups of HA4 was effected by reaction with only a moderate excess of $\mathrm{SO}_{3}$-pyridine complex and at $0{ }^{\circ} \mathrm{C}$ yielding mono- and disulfated products 1s-HA4- and 2s-HA4azide, which could be isolated by ion-exchange chromatography (Alshanski et al. 2019; Köhling et al. 2019). For the selective 4,6-protection of the $\mathrm{N}$-acetyl-glucosamine fragments, phenylboronates proved to be the suitable protecting groups. Completion of the protection step was monitored by ${ }^{1} \mathrm{H}$ NMR spectroscopy and the intermediary bis-boronates were efficiently converted into the pentasulfate 5s-HA4-azide. In the process, all cyclic boronates were cleaved with water without harnessing the five sulfate esters. Selective protection of the primary alcohols was achieved with dimethoxytrityl (DMT) chloride, allowing for the sulfation reaction yielding the hepta-sulfated 7s-HA4-azide, which was obtained without sulfate loss by hydrogenolytic cleavage of the DMT residue. Finally, conditions for the sulfation of all hydroxy groups were devised furnishing the pentasulfated 5s-HA2-azide, the

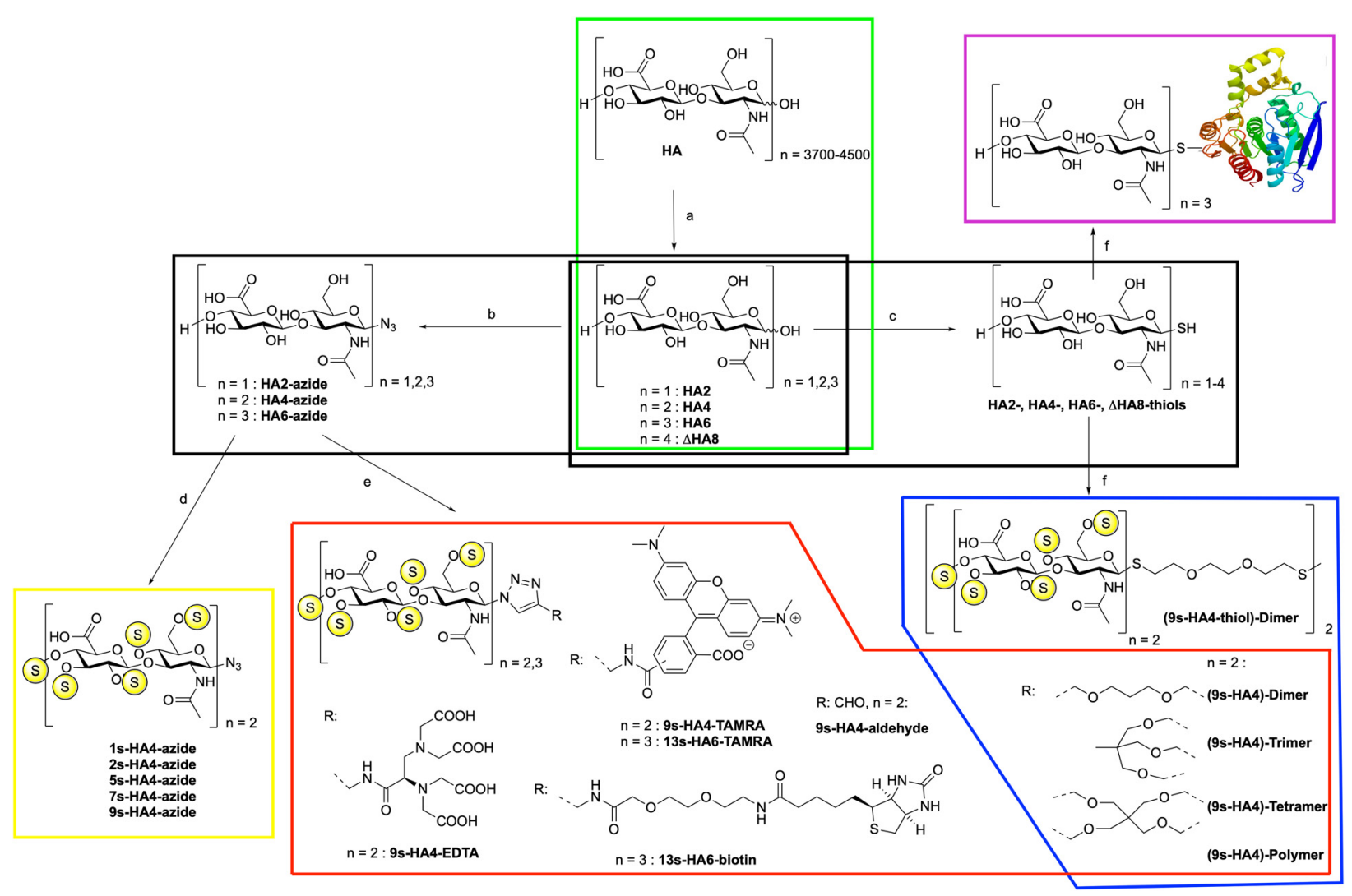

Figure 1: Preparation of defined sulfated GAG with anomeric modifications.

(a) Enzymatic digestion of hyaluronic acid (HA) to oligohyaluronanes (green). (b) Anomeric fixation to HA2,4,6-azides (black). (c) Anomeric fixation to HA2,4,6- and $\triangle$ HA8-thiols (black). (d) Defined sulfated GAG through selective protection/deprotection sequences (yellow). (e) Anomeric modification of GAG-azides through $\mathrm{Cu}(\mathrm{I}$ )-catalyzed Click reactions yielding EDTA-, TAMRA-, aldehyde, and biotin-labeled GAG (red) as well as multivalent dendritic und polymeric GAG (blue). (f) Click reactions of GAG-thiols to multivalent GAG architectures (blue) and GAG-labeled proteins (purple). 
nonasulfated 9s-HA4-azide, and the trideca-sulfated 13s-HA6-azide, respectively. All obtained defined sulfated GAG were obtained in preparative yields and fully characterized by fully assigned ${ }^{1} \mathrm{H}$ - and ${ }^{13} \mathrm{C}$-NMR spectra.

\section{Anomerically labeled GAG as chemical tools and their application}

Next, anomeric modifications were introduced for various applications and research projects (Figure 1(e)). First goal was the incorporation of a metal-chelating label in order to elucidate the structure of a GAG-complex with the exemplary protein interleukin 10 (IL-10) (Köhling et al. 2016a). HA4-azide was "clicked" with the EDTA-deri vative $\mathrm{N}$-propargyl-(2,2,3,3-tetra-methoxycarbonyl-methyl2R,3-diamino)-propanamide in the presence of $\mathrm{Cu}(\mathrm{I})$ ions and TBTA (Tris[(1-benzyl-1H-1,2,3-triazol-4-yl)methyl] amine) as a $\mathrm{Cu}(\mathrm{I})$ ligand. All methyl esters of the 1,2,3-triazol product were saponified and sulfation yielded the nonasulfate 9s-HA4-(triazolyl)-EDTA. The latter product enabled not only to quantify binding to IL-10 by chemical shift perturbations in HSQC-NMR spectra, but also to determine the precise position of the metalchelating, sulfated GAG, complexed with copper-(II) by using paramagnetic resonance enhancement (PRE) NMR on the protein surface and, thereby, to elucidate the structure of the IL-10-GAG complex.

\section{Fluorescent GAG}

An important contribution was the development of fluorophore-labeled sulfated GAG as these chemical tools enabled us to quantify GAG-protein binding with a collection of proteins (with only small amounts of protein required) and thereby gain new insights into GAG-protein binding (Figure 2). HA4- and HA6-azide, respectively, were reacted with 5,6- $\mathrm{N}$-propargylamido-tetramethylrhodamine furnishing the TAMRA-labeled HA4-(6-)(triazolyl)-TAMRA as a product, which was sulfated to yield 9s-HA4-TAMRA and 13s-HA6-TAMRA, respectively (Köhling et al. 2019). TAMRA-labeled sulfated GAG were used to establish competitive fluorescence polarization (FP) binding assays for 10 representative GAG-binding proteins, namely interleukins 8 and 10 (IL-8, IL-10), the growth factors bone morphogenic protein 2 (BMP2), transforming growth factor TGF-beta1, fibroblast growth factors FGF1 and FGF2, the chemokine CXCL-12, the Wntinhibitor sclerostin, and the protease inhibitors TIMP3 and antithrombin III (ATIII) (Figure 2(a)). Subsequently, the GAG-binding assays enabled us to investigate the structure-activity relationships for collections of defined sulfated and anomerically modified GAG (Figure 2(b)). Binding affinities determined in the FP assay were compared with those obtained from alternative quantitative binding assays, preferably NMR and isothermal titration calorimetry (ITC) (Figure 2(c)). This wealth of data generated a number of novel insights into GAG-protein binding, which, can be summarized here only in a short version: (1) GAG-binding sites recognize preferably tetra- or hexa-GAG. (2) Higher sulfation increases binding affinity. (3) Anomeric modification with a large hydrophobic (aromatic) residue increases drastically the binding affinities. (4) Alternative binding assays report different binding affinities. (5) Sulfated GAG bind to proteins at multiple binding sites and in multiple orientations (Figure 2(d)), thereby leading to a conclusive explanation for insight (4). These insights create a rational basis for the design of more specific GAG with increased affinity as further investigated below.

\section{GAG-biotin}

In order to characterize GAG-protein interactions in complex systems such as cells and cell lysates, a biotinylated sulfated GAG was developed. For this, (+)-biotin attached to a PEG-linker carrying a terminal alkyne residue was "clicked" to 13s-HA6-azide yielding 13s-HA6-biotin (Großkopf et al. submitted for publication). Subsequently, this product was added to cell lysates. After incubation, streptavidin beads were employed to pull-down all proteins binding directly or indirectly to the 13s-HA6 residue. After washing to remove non-specific binders, specific GAG-binding proteins were released from the streptavidin beads and cleaved proteolytically using the protease trypsin. Finally, interacting proteins were identified by LC-MS/MS analysis of the peptides obtained from the tryptic digest. Results and experimental details of this investigation will be published in due course.

\section{Multivalent GAG architectures}

Native GAG architectures in the extracellular matrix are typically characterized by the repetitive and branched presentation of multiple protein binding motifs. Thereby inspired, we investigated the binding of proteins to multivalent GAG architectures. A PEG-linked dimer of 9s-HA4-thiol (Figure 1(f)) displayed a strongly enhanced binding affinity to most of the 10 reference proteins using the fluorescence polarization assay (Köhling et al. 2019). This prompted us to initiate a systematic investigation of 
A. GAG-protein titration

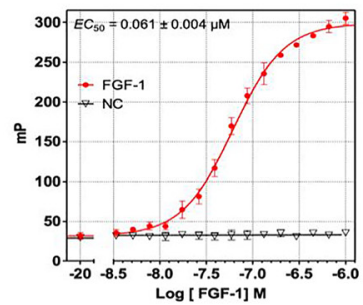

B. Competition assay

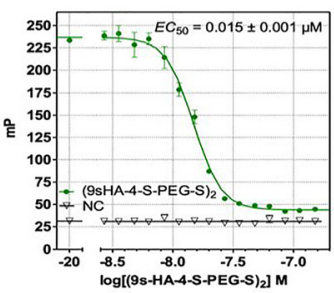

C. Thermodynamics
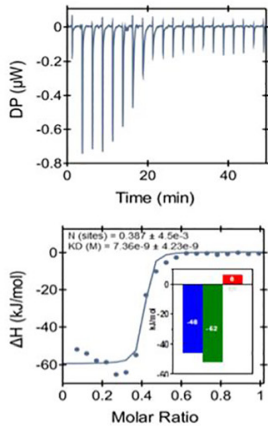

D. Diversity of GAG binding

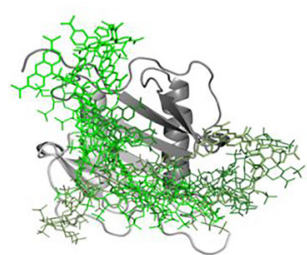

E. Anomeric fragment ligation

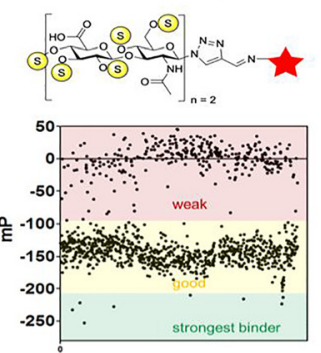

F. Specific, tailored GAG ligands

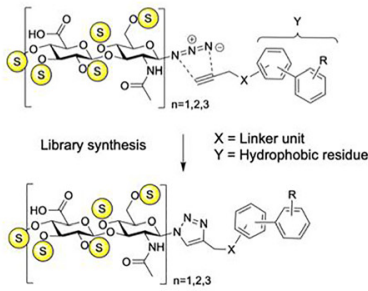

Figure 2: Insights into GAG-protein binding provide strategies for the development of protein-specific GAG ligands (Köhling et al. 2019). (a) Fluorescence polarization (FP) assays were established for 10 representative GAG-binding protein with 9s-HA4-TAMRA. (b) Collection of defined sulfated GAG validated in FP-competition assays. (c) Thermodynamics of GAG-protein interactions for selected monomeric and multimeric GAG ligands determined by isothermal calorimetry. (d) Results rationalized by the diversity of GAG binding sites, binding orientations and the impact of anomeric substituents. (e) Screening a library of 1152 amine fragments using the FP assay with interleukin 8 and the GAG

9s-HA4-aldehyde. (f) Development of specific, tailored GAG ligands for Wnt inhibitors using optimized GAG and anomeric fragment combinations.

[Correction added after online publication 22 July 2021: Figure 2 panel (f) was replaced by the current image, and legend text for Figure $2 f$ was changed.]

multivalent GAG architectures comparing the binding of 9s-HA-4-azide (and also methylthiol) to IL-8 protein with that of dimeric, trimeric, tetrameric and polymeric presentations of the monomer (Figure 1(e)). Binding to IL-8 was first investigated by fluorescence polarization and subsequently by isothermal titration calorimetry. Multivalent presentation of GAG monomer increased the binding affinity strongly down to nanomolar $K_{D}$-values. At the same time a pronounced crowding effect was observed especially with the rigidly connected GA (Köhling et al. 2019; Petitou et al. 1987) G-oligomers as indicated by strong deviations of the recorded thermograms from the one-to-one binding model and by reduced binding stoichiometries. These deviations were observed for the dimerizing wild-type IL-8, however, disappeared if a monomeric IL-8 mutant was employed. In contrast, oligomeric presentation of GAG-monomers on a flexible dextran polymer resulted in reduced binding affinities due to strongly negative binding entropy.

\section{On the road to protein-specific GAG ligands}

The strong impact of anomeric substituents on the binding affinity and specificity of GAG and especially the strong amplification exerted by large hydrophobic groups suggested that it might be possible to develop potent and specific ligands for target proteins. 9s-HA4-(triazolyl)aldehyde was developed starting from 9s-HA4 azide in order to enable the flexible variation of the residue in the anomeric position employing dynamic ligation screening (Figure 2(e)). In this assay, the aromatic aldehyde containing the 9s-HA4-motif was incubated with a model target protein, IL-8, the fluorescent ligand 9s-HA4-TAMRA, and with one nucleophilic amine fragment per microtiter plate well. Using fluorescence polarization as a read-out, a library of 1152 primary amines was screened obtaining several hits, which inhibited strongly the binding of the fluorescent probe. Best fragment-GAG combinations were re-synthesized and validated to confirm the amplification of binding. Details of these works will be reported at due course elsewhere.

Switching to a target protein with high functional relevance in the healing of bone tissue, protein-specific GAG ligands are being developed for Wnt-inhibitors like sclerostin and Dickkopf 1 (DKK-1) in a collaboration of chemical synthesis with molecular modelling and biological investigation (Figure 2(f)). For this purpose, various sulfated oligohyaluronane-azides were coupled with a set of alkynemodified hydrophobic fragments. The best, tailored GAG are currently under investigation in an animal model for bone 
repair, and the results of these works will be reported at due course.

[Correction added after online publication 22 July 2021: text of the last paragraph of the section "On the road to proteinspecific GAG ligands” was changed.]

\section{Cellular studies using defined sulfated GAG}

Defined sulfated GAG oligosaccharides were subsequently employed in functional biological studies with living cells and in animal models. For example, the molecules have been investigated in the inhibition of tissue inhibitor of matrix metalloprotease 3 (TIMP3) demonstrating a positive effect on bone healing (Rother et al. 2016a,b, 2017). Furthermore, the effect of oligomeric GAG on the signaling of vascular endothelial growth factor (VEGF) (Koehler et al. 2017, 2019; Rother et al. 2017) and of transforming growth factor beta-1 (TGF- $\beta 1$ ) and the implications for wound healing have been investigated. The molecules have been shown to improve wound healing through the release of heparinbinding epidermal growth factor (EGF) (Thönes et al. 2019) and were employed to develop mutant CXCL12 (Panitz et al. 2016; Spiller et al. 2019) with improved chemotactic properties through altering GAG binding and surface coating for the increased release of wound-healing cytokines (Clauder et al. 2020). Further studies on biomaterials for the targeted release of wound-healing protein factors are under way.

\section{Methods to characterize (sulfated) GAG oligosaccharides}

As outlined in another paper of this special issue by Schnabelrauch and coworkers, the characterization of GAG polysaccharides is very challenging because the application of mass spectrometry (MS) is nearly impossible and NMR is limited due to the broad resonances. Thus, only the chromatographically-determined molecular weight and the overall sulfate content is normally used to characterize GAG polysaccharides, whereas the detailed information at which carbon atom the individual sulfate residues are located is scarcely available (Schnabelrauch et al. 2013). The (gentle) fragmentation of the polysaccharides into oligosaccharides would be a reasonable method to overcome this problem. However, this approach is aggravated by the fact that artificial, oversulfated GAG cannot be enzymatically digested because the required enzymes are inhibited at these conditions (Lemmnitzer et al. 2014). Although there are many chemical methods of GAG depolymerization, these approaches are regularly accompanied by unwanted cleavage of the sulfate and/or the $\mathrm{N}$-acetyl residues. The method of "autohydrolysis" is timeconsuming and provides often only small yields of the oligosaccharides (Anastyuk et al. 2010). Therefore, the optimum way to synthesize oversulfated GAG oligosaccharides is to depolymerize the native polysaccharide (particularly hyaluronan (HA)) and subsequently use the obtained oligosaccharides for (chemical) sulfation (Köhling et al. 2019). Nevertheless, the question remains how the native oligosaccharides and particularly the oversulfated derivatives can be adequately and routinely characterized.

\section{The hyaluronan tetrasaccharide (HA4) as the starting material}

High molecular weight HA can be depolymerized by using the relatively inexpensive enzyme hyaluronidase from bovine testes (Köhling et al. 2016b). The cleavage mechanism of this enzyme is complex because the enzyme has both lyase and ligase activity, i.e. previously generated HA4 and HA2 are combined to HA6, two HA4 molecules to HA8 and so on. The fact that exclusively compounds with an even number of carbohydrate residues are generated, emphasizes the different stabilities of the $1 \rightarrow 3$ and the $1 \rightarrow 4$ glycosidic linkages. Anyway, a mixture of different oligosaccharides is generated, if polymeric HA is enzymatically digested: the yield of the tetrasaccharide is most significant followed by the hexasaccharide. This is the reason why we will focus here on HA4. The analytical separation of HA4 from the other digestion products is very simple and can be performed by virtually all established chromatographic methods including HPLC, column chromatography and electrophoresis (Šimek et al. 2020).

\section{Mass spectrometry}

It is well known that the HA oligosaccharides are detected with different sensitivities by MS (Schnabelrauch et al. 2013). Since MALDI-TOF (matrix-assisted laser desorption and ionization time-of-flight) MS is a desorption technique, it can be easily combined with thin-layer chromatography (TLC) and the developed TLC plates can be directly loaded (subsequent to the matrix application) into the mass spectrometer (Nimptsch et al. 2010). The negative ion MALDI-TOF mass spectra of selected GAG detected directly on a TLC plate are shown in Figure 3.

Although this method can be easily implemented at virtually all MALDI mass spectrometers, there are two 


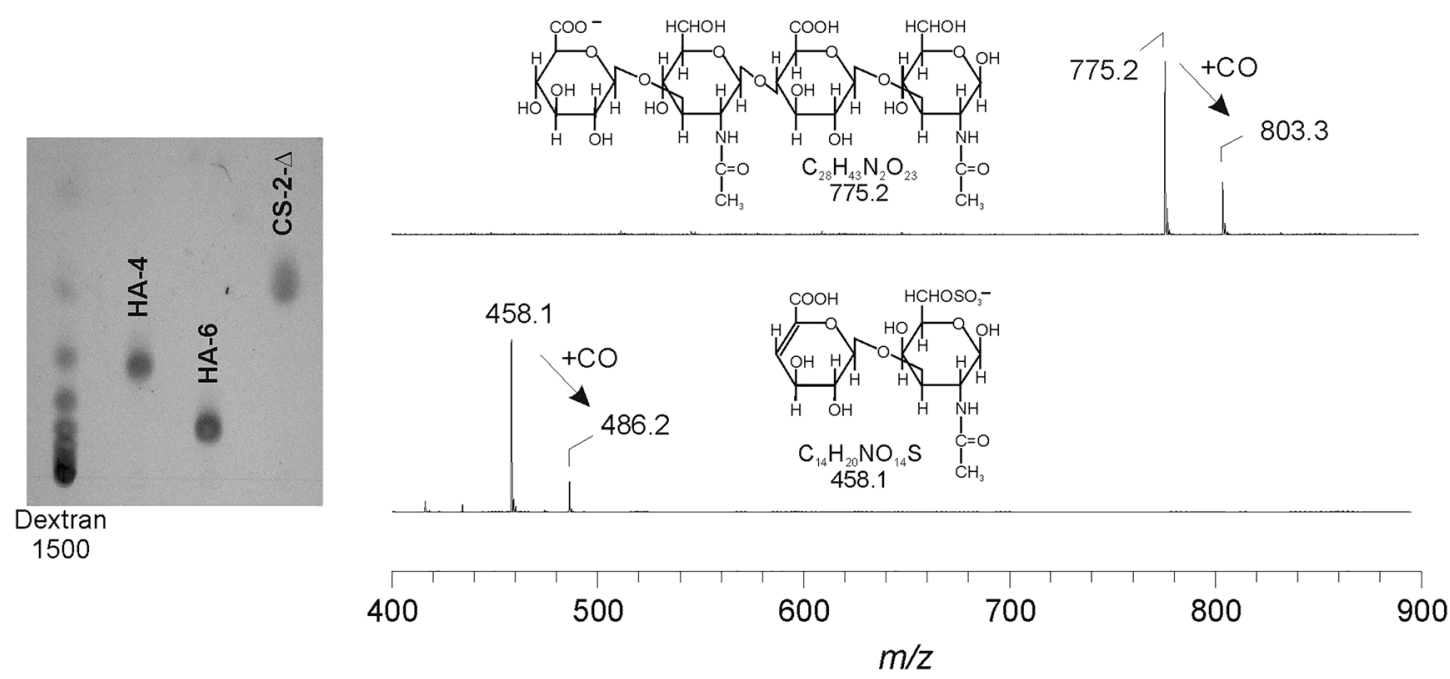

Figure 3: Negative ion MALDI-TOF mass spectra of HA4 (upper spectrum) and the unsaturated disaccharide of chondroitin sulfate (lower spectrum).

Spectra were recorded directly from the TLC plate (shown at the left). The structures and the expected $m / z$ ratios are provided along with the mass spectra. Please note that "+CO" indicates the formation of the formyl ester because the separation of the carbohydrates was performed in the presence of formic acid. Dextran 1500 as well as HA6 are only shown for comparative purposes on the TLC plate (left) but no mass spectra of these compounds are provided. Reprinted with modification and permission (from Elsevier) from (Nimptsch et al. 2010).

problems that complicate the situation: the first problem is coming from the use of high concentrations of formic acid that are required to be able to separate GAG on a normal phase TLC plate (Zhang et al. 2007). Under these conditions, esterification of selected hydroxyl groups occurs (detectable by the characteristic mass shift of 28 mass units) and, thus, a moiety of the GAG is not detectable as such but only as the corresponding formyl ester. A second, more serious problem is the loss of sulfate residues. This does, however, occur under (at least partially) all the so far tested MALDI MS conditions and this loss is only slightly enhanced if the spectra are acquired directly on the TLC plate. Although the positive ion mode is much less sensitive, this polarity mode suffers to a lesser extent from the sulfate loss (Nimptsch et al. 2012) and should, thus, preferentially be used. Another method might be the use of ionic liquid matrices (Petković et al. 2020).

The number of counterions is extremely important if ESI (electrospray ionization) MS is used and the drug "Fondaparinux" (available as solution in physiological saline) was employed to optimize the acquisition conditions (Lemmnitzer et al. 2021). It is obvious that the spectral quality depends extremely on the applied conditions (cf. Figure 4) and careful fine-tuning is necessary.

Two desalination protocols were evaluated: cation exchange with triethylammonium (TEA) counterions and size-exclusion chromatography (SEC). In each case the intensities of the most abundant intact analyte ion species were monitored in the positive and the negative ion mode observing a large mass range between 80 and $2200 \mathrm{~m} / \mathrm{z}$ (pos and neg) as well as for the positive ion mode using a restricted range, i.e. between 400 and $2200 \mathrm{~m} / z$ (pos 2). Cation exchange without neutralization is not shown because exclusively unwanted fragment ions were detected independent of the polarity. The sample concentration was varied between $1 \mu \mathrm{mol} \mathrm{L}^{-1}$ and $1 \mathrm{mmol} \mathrm{L}^{-1}$.

It is also important that the process of ESI MS leads to a considerable decrease in the $\mathrm{pH}$ of the considered solutions. This should be considered if odd carbohydrates are detected. These species are not generated enzymatically but generated as an artifact by the MS conditions (Prebyl et al. 2003).

\section{NMR spectroscopy}

NMR is an important tool to study oligosaccharides since the most serious problem of NMR-based analysis, the poor sensitivity, was overcome by the invention of inverse methods, i.e. insensitive nuclei such as ${ }^{13} \mathrm{C}$ or ${ }^{15} \mathrm{~N}$ are indirectly detected via the much higher sensitivity of ${ }^{1} \mathrm{H}$. This is particularly important if complex (and, thus, timeconsuming) pulse sequences are used (Soares et al. 2017). Although the resonances of the anomeric protons are normally well resolved, it is difficult to discriminate similar compounds (such as HA4 and HA6) by standard NMR. Pulsed field-gradient (PFG) NMR measures the diffusion behavior of a given compound in solution and this parameter reflects even small changes of the molecular 
(a)ESI IT mass spectra of Fondaparinux

(1) after cation exchange (w/o neutralization)
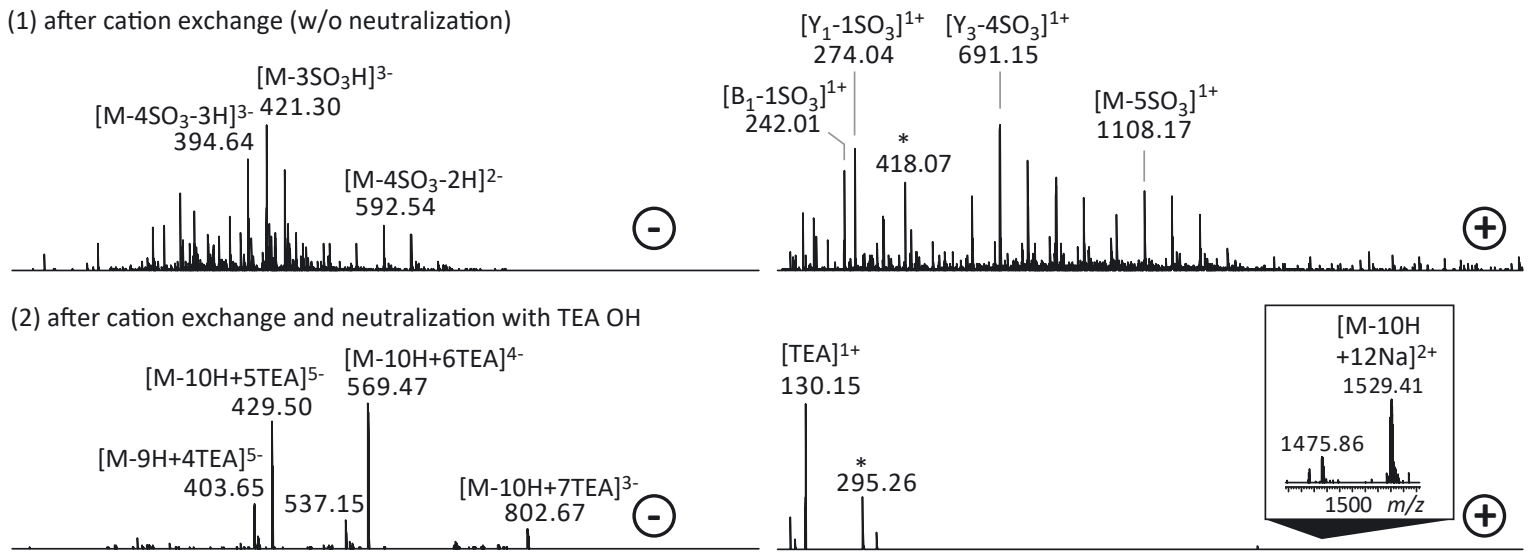

(2) after cation exchange and neutralization with TEA OH

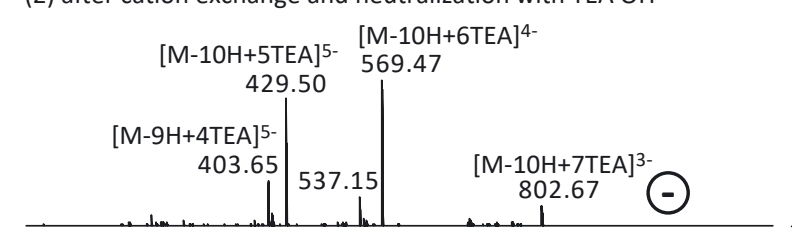

(3) after size-exclusion chromatography $[\mathrm{M}-8 \mathrm{H}+4 \mathrm{Na}]^{4-}$
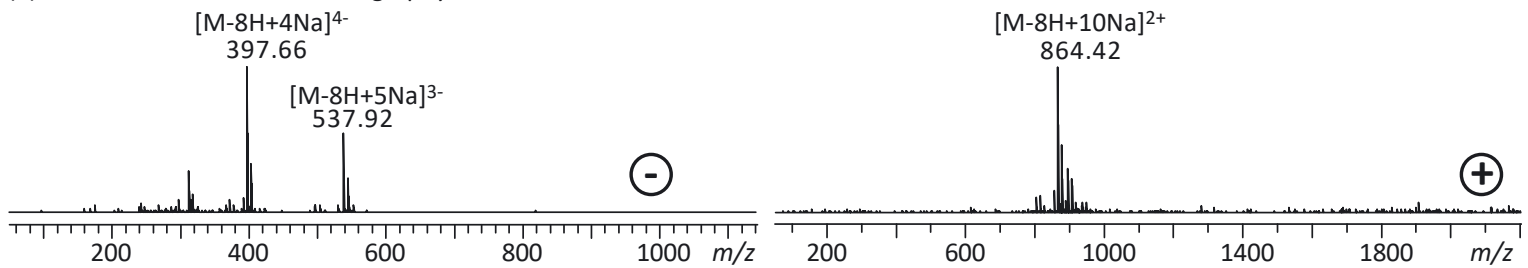

\section{(b) Chemical structure of Fondaparinux}

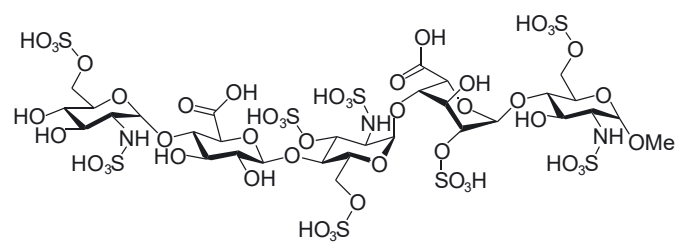

(c) Intensities of the most abundant intact ion peaks of fondaparinux

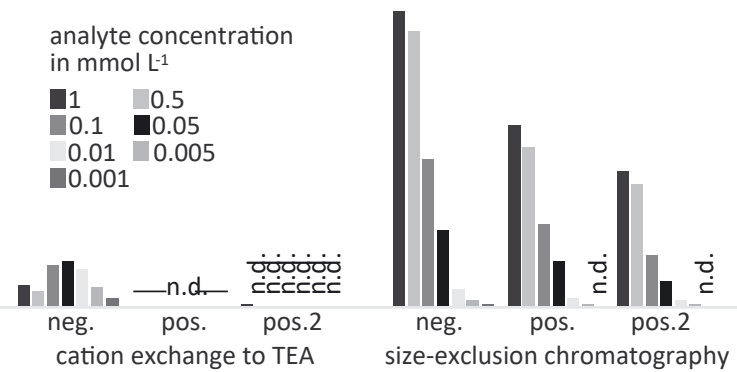

Figure 4: Electrospray ion trap mass spectra of Fondaparinux.

(a) ESI mass spectra of selected Fondaparinux samples after different desalination protocols. The ESI IT mass spectra of fondaparinux after three different desalination protocols in both polarities (+, -): cation exchange without neutralization (1) leads to sulfate loss and cleavage of the glycosidic linkages; neutralization with triethylamine (TEA) (2) afterwards allows the detection of intact analyte ions with a minimized loss of sulfate groups. However, this contaminates the instrument and affects the detection limits particularly in the positive ion mode; desalination via size-exclusion (3) gave the best results. The sample concentration for the measurements was $1 \mathrm{mmol} \mathrm{L}^{-1}$. Reprinted with modifications and permission from Wiley (Lemmnitzer et al. 2021). (b) Chemical structure of Fondaparinux D-GIcNS6S- $\alpha$-(1,4)-D-GIcA- $\beta-(1,4)$ D-GIcNS3,6S- $\alpha$-(1,4)-L-IdoA2S- $\alpha$-(1,4)-D-GlcNS6S-OMe; $M_{\mathrm{Fpx}}=1506.95$ (monoisotopic). (c) Intensities of the most abundant intact ion peaks of fondaparinux in the ESI mass spectra. n.d. = not detected.

size - as well as in the case of overlapping resonances. If a two-dimensional experiment is performed, this method is well-known as "DOSY" (diffusion-ordered spectroscopy). Using PFG ${ }^{1} \mathrm{H}$ NMR it is also possible to characterize isomeric carbohydrates such as the 4- and 6-sulfate of the disaccharide of chondroitin sulfate (CS) (Lemmnitzer et al. 2016). Unfortunately, the introduction of additional sulfate residues in GAG oligosaccharides reduces the frequency dispersion making assignments from the ${ }^{1} \mathrm{H}$ NMR spectra difficult. One old, but powerful technique to determine whether there is an $-\mathrm{OH}$ group or a $-\mathrm{O}-\mathrm{SO}_{3} \mathrm{H}$ at a dedicated position relies on $\mathrm{H} / \mathrm{D}$ exchange. The spectrum is acquired in $\mathrm{H}_{2} \mathrm{O}$ and subsequently in $\mathrm{D}_{2} \mathrm{O}$. If there is no change in the chemical shift there is a sulfate residue at the respective carbon atom, otherwise there is a free hydroxyl group. Finally, NMR is the method of choice to elucidate the binding of a GAG to a dedicated protein such as growth factors, hormones, cytokines, or chemokines. This is normally performed by recording the ${ }^{15} \mathrm{~N}$ NMR spectra of the protein in the absence and the presence of the GAG. 
Interactions are reflected by changes of the chemical shift of the respective amino acid resonances NMR data are often complemented with molecular modeling data since computational chemistry is capable of predicting potential binding sites between proteins and GAG. These predictions can be used to focus on the synthesis of selected GAG that are particularly promising.

\section{Infrared and Raman spectroscopy}

Although the sulfate group is strongly polarized and should be, thus, easily detectable by means of infrared (IR) spectroscopy, there are only a few publications available where the potential of IR and Raman was shown. More recent studies were performed with oligo- not polysaccharides (Lettow et al. 2020).

\section{Summary and outlook}

This review was aimed to provide a concise survey about (a) chemical methods to generate GAG oligosaccharides with defined sulfation patterns, (b) suitable approaches to analyze the obtained GAG derivatives and (c) the discussion of the interactions between these GAG and proteins with significant in vivo relevance. Hopefully, we were able to show that chemically modified, oversulfated GAG derivatives exhibit a multitude of interesting effects and are, thus, not only of interest in basic sciences but as well in regenerative medicine. Although the chemical modification of GAG is still a challenging task due to the need of regioselective reactions and the introduction as well as the removal of protecting groups, these problems can be expected to be overcome in the future. This paves the way for broader applications of these compounds. Nevertheless, it must be emphasized that the characterization of these compounds is extremely important - in particular if the biological effects are of interest. Poorly characterized materials may be one reason why inconsistent data of the related biological effects are reported.

Author contributions: All the authors have accepted responsibility for the entire content of this submitted manuscript and approved submission.

Research funding: The work presented in this review was funded by the Deutsche Forschungsgemeinschaft (DFG, project number 59307082 - TRR67, subproject A8).

Conflict of interest statement: The authors declare no conflicts of interest regarding this article.

\section{References}

Alshanski, I., Blaszkiewicz, J., Mervinetsky, E., Rademann, J., Yitzchaik, S., and Hurevich, M. (2019). Sulfation patterns of saccharides and heavy metal ions binding. Chem. Eur J. 25: 12083-12090.

Anastyuk, S.D., Shevchenko, N.M., Nazarenko, E.L., Imbs, T.I., Gorbach, V.I., Dmitrenok, P.S., and Zvyagintseva, T.N. (2010). Structural analysis of a highly sulfated fucan from the brown alga Laminaria cichorioides by tandem MALDI and ESI mass spectrometry. Carbohydr. Res. 345: 2206-22012.

Clauder, F., Möller, S., Köhling, S., Bellmann-Sickert, K., Rademann, J., Schnabelrauch, M., and Beck-Sickinger, A.G. (2020). Peptidemediated surface coatings for the release of wound-healing cytokines. J. Tissue Eng. Regen. Med. 14: 1738-1748.

Eller, S., Collot, M., Yin, J., Hahmm, H.S., and Seeberger, P.H. (2013). Automated solid-phase synthesis of chondroitin sulfate glycosaminoglycans. Angew. Chem. Int. Ed. 52: 5858-5861.

Exner, M.P., Köhling, S., Rivollier, J., Gosling, S., Srivastava, P., Palyancheva, Z.I., Herdewijn, P., Heck, M.P., Rademann, J., and Budisa, N. (2016). Incorporation of amino acids with long-chain terminal olefins into proteins. Molecules 21: 287-295.

Großkopf, H., Vogel, S., Damaris Müller, C., Köhling, S., Dürig, J.-N., Möller, S., Schnabelrauch, M., Rademann, J., Hempel, U., von Bergen, M., et al. (2021 Submitted for publication). Identification of intracellular glycosaminoglycan-interacting proteins by affinity purification mass spectrometry. Biol. Chem.

Koehler, L., Samsonov, S., Rother, S., Vogel, S., Köhling, S., Moeller, S., Schnabelrauch, M., Rademann, J., Hempel, U., Pisabarro, M.T., et al. (2017). Sulfated hyaluronan derivatives modulate TGF$\beta 1$ : receptor complex formation: possible consequences for TGF$\beta 1$ signaling. Sci. Rep. 7: 1210.

Koehler, L., Ruiz-Gómez, G., Balamurugan, K., Rother, S., Freyse, J., Möller, S., Schnabelrauch, M., Köhling, S., Djordjevic, S., Scharnweber, D., et al. (2019). Dual action of sulfated hyaluronan on angiogenic processes in relation to vascular endothelial growth factor-A. Sci. Rep. 9: 18143.

Köhling, S., Künze, G., Lemmnitzer, K., Bermudez, M., Wolber, G., Schiller, J., Huster, D., and Rademann, J. (2016a).

Chemoenzymatic synthesis of nonasulfated tetrahyaluronan with a paramagnetic tag for studying its complex with interleukin-10. Chem. Eur J. 22: 5563-5574.

Köhling, S., Blaszkiewicz, J., Ruiz-Gómez, G., Fernández-Bachiller, M.I., Lemmnitzer, K., Panitz, N., Beck-Sickinger, A.G., Schiller, J., Pisabarro, M.T., and Rademann, J. (2019). Syntheses of defined sulfated oligohyaluronans reveal structural effects, diversity and thermodynamics of GAG-protein binding. Chem. Sci. 10: 866-878.

Köhling, S., Exner, M.P., Nojoumi, S., Schiller, J., Budisa, N., and Rademann, J. (2016b). One-pot synthesis of anomeric glycosyl thiols from highly functionalized unprotected sugars in water for use in glycan ligation reactions. Angew. Chem. Int. Ed. 55: 15510-15514.

Lemmnitzer, K., Köhling, S., Freyse, J., Rademann, J., and Schiller, J. (2021). Characterization of defined sulfated heparin-like oligosaccharides by electrospray ionization ion trap (ESI IT) mass spectrometry. J. Mass Spectrom. 56: e4692.

Lemmnitzer, K., Riemer, T., Groessl, M., Süß, R., Knochenmuss, R., and Schiller, J. (2016). Differentiation of chondroitin sulfate 
isomers by IMS mass spectrometry and NMR spectroscopic diffusion measurements. Anal. Methods 8: 8483-8491.

Lemmnitzer, K., Schiller, J., Becher, J., Möller, S., and Schnabelrauch, M. (2014). Improvement of the digestibility of sulfated hyaluronans by bovine testicular hyaluronidase: a UV spectroscopic and mass spectrometric study. BioMed Res. Int. 2014: 986594.

Lettow, M., Grabarics, M., Mucha, E., Thomas, D.A., Polewski, t., Freyse, J., Rademann, J., Meijer, G., von Helden, G., and Pagel, K. (2020). IR action spectroscopy of glycosaminoglycan oligosaccharides. Anal. Bioanal. Chem. 412: 533-537.

Nimptsch, K., Süss, R., Riemer, T., Nimptsch, A., Schnabelrauch, M., and Schiller, J. (2010). Differently complex oligosaccharides can be easily identified by matrix-assisted laser desorption and ionization time-of-flight mass spectrometry directly from a standard thinlayer chromatography plate. J. Chromatogr. A 1217: 3711-3715.

Nimptsch, K., Süß, R., Schnabelrauch, M., Nimptsch, A., and Schiller, J. (2012). Positive ion MALDI-TOF mass spectra are more suitable than negative ion spectra to characterize sulphated glycosaminoglycans. Int. J. Mass Spectrom. 310: 72-76.

Panitz, N., Theisgen, S., Samsonov, S.A., Gehrcke, J.P., Baumann, L., Bellmann-Sickert, K., Köhling, S., Pisabarro, M.T., Rademann, J., Huster, D., et al. (2016). The structural investigation of glycosaminoglycan binding to CXCL12 displays distinct interaction sites. Glycobiology 26: 1209-1221.

Petitou, M., Duchaussoy, P., Lederman, I., Choay, J., Jacquinet, J.C., Sinaÿ, P., and Torri, G. (1987). Synthesis of heparin fragments: a methyl alpha-pentaoside with high affinity for antithrombin III. Carbohydr. Res. 167: 67-75.

Petković, M., Leopold, J., Popović, I., Dimić, D., Ilić, J., Nenadović, M., Rakočević, Z., and Schiller, J. (2020). Performances of ionic liquid matrices with butyl ammonium counterion for matrix-assisted laser desorption/ionization mass spectrometric detection and analysis of sucralfate. J. Carbohydr. Chem. 39: 1-23.

Prebyl, B.S., Kaczmarek, C., Tuinman, A.A., and Baker, D.C. (2003). Characterizing the electrospray-ionization mass spectral fragmentation pattern of enzymatically derived hyaluronic acid oligomers. Carbohydr. Res. 338: 1381-1387.

Rother, S., Samsonov, S.A., Hofmann, T., Blaszkiewicz, J., Köhling, S., Moeller, S., Schnabelrauch, M., Rademann, J., Kalkhof, S., von Bergen, M., et al. (2016a). Structural and functional insights into the interaction of sulfated glycosaminoglycans with tissue inhibitor of metalloproteinase-3 - a possible regulatory role on extracellular matrix homeostasis. Acta Biomater. 45: 143-154.

Rother, S., Samsonov, S.A., Hempel, U., Vogel, S., Moeller, S., Blaszkiewicz, J., Köhling, S., Schnabelrauch, M., Rademann, J., Pisabarro, M.T., et al. (2016b). Sulfated hyaluronan alters the interaction profile of TIMP-3 with the endocytic receptor LRP-1 clusters II and IV and increases the extracellular TIMP-3 level of human bone marrow stromal cells. Biomacromolecules 17: 3252-3261.

Rother, S., Samsonov, S.A., Moeller, S., Schnabelrauch, M., Rademann, J., Blaszkiewicz, J., Köhling, S., Waltenberger, J., Pisabarro, M.T., Scharnweber, D., et al. (2017). Sulfated hyaluronan alters endothelial cell activation in vitro by controlling the biological activity of the angiogenic factors vascular endothelial growth factor-A and tissue inhibitor of metalloproteinase-3. ACS Appl. Mater. Interfaces 9: 9539-9550.

Schnabelrauch, M., Scharnweber, D., and Schiller, J. (2013). Sulfated glycosaminoglycans as promising artificial extracellular matrix components to improve the regeneration of tissues. Curr. Med. Chem. 20: 2501-2523.

Šimek, M., Lemr, K., Hermannová, M., and Havlíček, V. (2020). Analysis of hyaluronan and its derivatives using chromatographic and mass spectrometric techniques. Carbohydr. Polym. 250: 117014.

Soares, P.A., Queiroz, I.N., and Pomin, V.H. (2017). NMR structural biology of sulfated glycans. J. Biomol. Struct. Dyn. 35: 1069-1084.

Spiller, S., Panitz, N., Limasale, Y.D.P., Atallah, P.M., Schirmer, L., Bellmann-Sickert, K., Blaszkiewicz, J., Köhling, S., Freudenberg, U., Rademann, J., et al. (2019). Modulation of human CXCL12 binding properties to glycosaminoglycans to enhance chemotactic gradients. ACS Biomater. Sci. Eng. 5: 5128-5138.

Tanaka, T., Nagai, H., Noguchi, M., Kobayashi, A., and Shoda, S. (2009). One-step conversion of unprotected sugars to $\beta$-glycosyl azides using 2-chloroimidazolinium salt in aqueous solution. Chem. Commun. 3378-3379, https://doi.org/10.1039/ b905761g.

Thönes, S., Rother, S., Wippold, T., Blaszkiewicz, J., Balamurugan, K., Moeller, S., Ruiz-Gómez, G., Schnabelrauch, M., Scharnweber, D., Saalbach, A., et al. (2019). Hyaluronan/collagen hydrogels containing sulfated hyaluronan improve wound healing by sustained release of heparin-binding EGF-like growth factor. Acta Biomater. 86: 135-147.

Vibert, A., Lopin-Bon, C., and Jacquinet, J.-C. (2009). From polymer to size-defined oligomers: a step economy process for the efficient and stereo-controlled construction of chondroitin oligosaccharides and biotinylated conjugates thereof: part 1. Chem. Eur J. 15: 9561-9578.

Zhang, Z., Xie, J., Zhang, F., and Linhardt, R.J. (2007). Thin-layer chromatography for the analysis of glycosaminoglycan oligosaccharides. Anal. Biochem. 371: 118-120. 